Research Article

\title{
A Tripartite Evolutionary Game Analysis of Enterprises' Behaviour in the Platform Ecosystem
}

\author{
Ying Han \\ School of Economics, Fujian Normal University, Fuzhou 350117, China \\ Correspondence should be addressed to Ying Han; ying_han_@hotmail.com
}

Received 3 March 2020; Revised 14 June 2020; Accepted 23 June 2020; Published 3 August 2020

Academic Editor: Filippo Cacace

Copyright (c) 2020 Ying Han. This is an open access article distributed under the Creative Commons Attribution License, which permits unrestricted use, distribution, and reproduction in any medium, provided the original work is properly cited.

\begin{abstract}
With the rapid progress of digital technology and new business models' development, the platform ecosystem has not only penetrated every sector in society but also revealed various problems. Platform governance researchers have highlighted its significance, but failed to adequately consider ecosystem participants' behaviour. This paper aims to explore platform governance's effects on enterprises' behaviour in a multiorganisational platform ecosystem context. The tripartite evolutionary game is chosen to construct a mathematical model that integrates the government, platform organisation, and internal enterprises to analyse formal and informal governance's influence on enterprises' behaviour in the platform ecosystem. It was discovered that both formal and informal governance can decrease internal enterprises' opportunistic behaviours. Furthermore, formal governance has stronger effects than informal governance, although informal governance's effects are more stable. Additionally, selforganisation in the platform ecosystem is another important area of focus. This paper enriches an understanding of platform ecosystems while formulating insights relevant in using platform governance to develop small and medium-sized enterprises.
\end{abstract}

\section{Introduction}

More than four billion people are currently connected to the Internet, and this number is expected to increase to $70 \%$ of the world's population in 2020 [1]. The Internet can provide some companies with a way to experience the rise of platform $[2,3]$, an increasingly innovative communications channel. Platform organisations, such as Amazon, Etsy, Facebook, Airbnb, and Uber, allow multiple stakeholders with complementary functions to work closely and leverage their networks, ultimately creating "platform ecosystems" [4-7]. Thus, the rapid development of information technologies and the new business models supported by them have caused scholars from the different disciplines to focus on these platforms and their related ecosystems [8-10].

The "platform" is an intermediary that connects markets from different user groups and relies on technology and information to promote value-creating interactions $[11,12]$. Indirect or cross-network effects allow these platforms to create value between two different user groups $[8,9,13,14]$. The network effect emphasises platform ecosystems' importance [15], as the platform ecosystem can be perceived as a multiplatform metaorganisation-or a collection of organisations and individuals - with a common vision for the platform's prosperity $[16,17]$. The ecosystem concept highlights the nature of the interorganisational network, collaborations, and competition among its participants, and its high interdependence and ability to evolve with external environmental changes [18-20]. The platform ecosystem consists of multiple actors with joint value and coevolution relationships $[8,21]$, including stakeholders such as the platform organisation, government, small and mediumsized enterprises (SMEs), customers, research and development institutions, universities, financial institutions, and their intermediaries [22].

However, a vast majority of platform organisations are driven by commercial interests, and commercial interests are typically prioritised over social values [23]. Platform organisations tend to bypass the institutional processes through which societies are organised, such as legislation, sectoral regulations, or any public accountability or responsibility. Facebook, Google, and Uber, among other large platforms, 
have argued that they are merely "facilitators" to connect users to creators or producers, thus avoiding conventional legal categories. For example, Uber refused to accept its status as a "transport company" until the European Court of Justice ultimately confirmed this in December 2017 [23]. Taobao in particular has experienced a high volume of counterfeit sales, with several false car rental platforms and short-term rental service platforms that have caused safety issues; these have attracted public attention in China and worldwide [24]. Consequently, an important issue in platform ecosystems involves the realisation of effective platform governance.

The question of how platforms should be governed which has no simple answer as the policy arena is fragmented, with responsibility for the platform ecosystem's social and political roles divided among: governments, as the entity that establishes the overall rules for these interactions; the platform organisation, as architects of online environments; and internal enterprises or users, as SMEs or individuals that make decisions about their specific behaviours in online environments [25]. Existing literature discusses many types of governance mechanisms [26, 27]. One is formal or government-based, such as market contracts, intellectual property, and other legally binding agreements used as a basis for collective action and decision-making [28]. Another type of governance mechanism is informal or platform-based and depends on mutual trust, agreed codes of conduct, beliefs in a shared vision, and similar cultures [10]. Both formal and informal governance approaches exist in the platform ecosystem. For example, the norms of privacy, fairness, and access in educational platforms are controlled by a combination of governmental oversight and agreements between schools and parents [23]. Evidence also indicates that corporate governance in international platforms is derived from formal rules, such as government censorship, and informal rules, such as norms, culture, and value; both of these impact value-creation activities [11].

As the platform ecosystem clearly involves multiple participants, their interests can differ [29-31], and thus, the government must act as legislator, regulator, moderator, and executor to negotiate in the public's best interest [23]. Many governments have acted to restrain illegitimate platform organisations [32]. For example, the Indian government provided notice to platform-based online retailers to restrict any subsidiary or affiliated entity of the person in charge of the platform from selling on the platform and has prohibited any partner from obtaining discounts. Widespread speculation also exists that a similar government censorship system is intended to impact the performance of platform companies in China [11]. From a European perspective, all levels of government can and should actively negotiate on behalf of platform consumers [23]. Generally, these governments have the responsibility to provide guidance and benchmarks to evaluate how the platform fulfils its organisational responsibilities, standardise these enterprises' behaviour, and establish effective oversight and monitoring mechanisms [25].
In the platform ecosystem context, platform organisations must also create conditions to incentivise and influence the internal enterprises to achieve their desired results [33]. However, this informal or platform-based governance fundamentally differs from the formal or government-based model. Platform organisations typically do not officially authorise other partners, but loosely work with them $[34,35]$. The platform's generative nature enables unprecedented participants to work together and establish large, complex partner networks [36]. Platform organizations exercise various formal and informal control mechanisms to influence third-party participants' behaviour and resulting performance [37]. Nevertheless, the platform ecosystem consists of a highly dynamic, unstructured environment composed of thousands of participants, and its informal control mechanisms are more suitable for platform owners [34, 37]. Therefore, relative to formal governance, informal or platformbased governance encourages member enterprises to commit themselves to mutual beliefs and goals, which often compels them to engage in the proper behaviour and produce better performance outcomes based on shared values and norms. Informal or platform-based governance is particularly important when the required results and behaviours are unknown or difficult to monitor [37].

Various studies have highlighted the significance of governance in the platform ecosystem, but have failed to adequately consider the ecosystem participants' behaviour $[38,39]$. In the platform ecosystem, complementors are not constrained by platform organization [35, 40]. Therefore, due to the lack of contracts or hierarchical relationships, it is difficult for platform organizations to control their internal entities [37]. Additionally, an ecosystem may include many complementors, which requires more cost-effective governance mechanisms [41], such as government regulations, market or intellectual property contracts, and other legally binding agreements. As a result, both formal or government-based and informal or platform-based governance must be considered in the platform ecosystem context.

This paper aims to address these gaps by exploring informal or platform-based and formal or government-based governance in the context of multiorganisational platform ecosystems. We accomplish this through a tripartite evolutionary game to analyse the behaviour among ecosystem participants.

\section{Evolutionary Game Model}

2.1. Model Variables and Assumptions. A tripartite evolutionary game model that includes the government, platform organisations, and internal enterprises' behaviour is proposed based on the previous discussion as well as some assumptions.

Assumption 1. Platform organisations and internal enterprises should comply with local government laws; for example, platforms should follow the government's notice- 
and-takedown requests regarding content [42]. If enterprises in the platform demonstrate honest, trustworthy behaviours, the public will praise the government's supervisory behaviour. This will enhance the government's credibility and provide other social benefits, such as social stability and economic development. If enterprises exhibit negative behaviours, then the government must bear an indirect loss of social image and the external public pressure caused by this breach of trust [22, 43]. Similarly, if informal or platform-based governance is effective, it is obviously in the interest of the platform organisation, at least from a moral standpoint $[10,25]$. However, ineffective informal or platform-based governance might simultaneously have severe negative and indirect impacts on the platform's reputation and customer satisfaction $[10,37,44]$. The following model variables are proposed based on this assumption:

$E_{i}$ : social feedback of the government regarding internal enterprises' honest or dishonest behaviour $(i=1,2,3,4)$. When enterprises exhibit honest behaviour, this creates better social feedback for the government, and thus, $E_{1}, E_{2} \geq E_{3}, E_{4}$.

$R$ : the benefit of the platform.

$F_{i}$ : the benefit of internal enterprises regarding their honest or dishonest behaviours $(i=1,2,3,4)$. When the formal and informal governance is valid, enterprises exhibit honest behaviour; this creates more value for the enterprises, and thus, $F_{1}>F_{2}, F_{3}>F_{4}$.

Assumption 2. The government will incur the costs from offering formal governance, such as facilitating collective actions, providing tools, managing assets, and ensuring public value, among other regulatory activities [45-47]. The platform will also incur the costs to regulate its enterprises $[46,48,49]$. Additionally, enterprises assume production costs from daily operations and may attempt to reduce these costs through dishonest business transactions to gain opportunistic profits. The following model variables are proposed based on this assumption:

$C_{i}$ (costs): specifically, $C_{1}$ denotes the government's cost of supervising enterprises; $C_{2}$ is the government's cost to supervise the platform; $C_{3}$ is the platform organisation's regulatory costs; $C_{4}$ is the enterprise's operating costs; and $w C_{4}$ indicates dishonest enterprises' production costs, with $0<w<1$.

Assumption 3. If informal governance is effective, then the government will provide incentives to platform organisations. If enterprises operate in good faith, the government will also subsidise their operating costs $[11,23]$. However, if enterprises breach established conditions or regulations, they will be punished by both formal and informal governance, and the platform organisation will also face the government's punishment for absence of regulation [10]. The following model variables are proposed based on this assumption: $m$ : the government bonus to the platform organisation $n$ : the ratio of government subsidies to enterprises $p$ : the government's punishment for dishonest enterprises

$l$ : the ratio of government's punishment for the platform organisation

$q$ : the platform organisation's punishment for dishonest enterprises

Assumption 4. Multiple participants interact with each other in the platform ecosystem. Network externalities-such as the platform's number of end-users/complementors-will increase (decrease) when the value attached to the platform is higher (lower). Thus, platform organisations must exercise caution in their governance to allow the entire platform to develop [24]. Internal enterprises' honesty will also affect their platform's reputation. Similarly, whether the platform organisation has effective governance to build a better business environment will also affect the internal enterprises' reputation [10, 37, 44]. The following model variables are proposed based on this assumption:

$\lambda_{1}$ : a coefficient indicating the platform's reputation $\left(0<\lambda_{1}<1\right)$

$\lambda_{2}$ : a coefficient indicating the enterprises' reputation $\left(0<\lambda_{2}<1\right)$

2.2. Evolutionary Game Model. The government, platform, and enterprises belong to an evolutionary game group with finite rationality [50]. The previously discussed assumptions reveal that under valid formal governance, the government will pay the costs for its subsidies, and enterprises will be penalised for any dishonesty. If informal governance is effective, the platform organisation will benefit from an orderly market environment in addition to subsidised operating costs; they will also be able to obtain additional government incentives. However, if informal governance is invalid and internal enterprises exhibit opportunistic behaviour, the government will punish the platform organisation. In addition to paying their own operating costs, internal enterprises can earn government subsidies through good faith behaviour. In contrast, dishonesty will result in a dual punishment from both the government and the platform organisation.

Given this discussion and the previously mentioned assumptions, a matrix of the tripartite evolution game's benefits in the platform ecosystem can be obtained, as displayed in Tables 1 and 2, which provide the specific definitions for each side.

Suppose the probability that the government chooses the "valid formal governance" strategy is $x$ and the probability of choosing the "invalid formal governance" strategy is $(1-x)$; suppose the probability that the platform chooses the "valid 
TABLE 1: The payoff matrix for the game's three sides.

\begin{tabular}{|c|c|c|c|c|}
\hline \multirow[b]{2}{*}{ Enterprises } & \multicolumn{2}{|c|}{ Valid formal governance $(x)$} & \multicolumn{2}{|c|}{ Invalid formal governance $(1-x)$} \\
\hline & $\begin{array}{l}\text { Valid informal } \\
\text { governance }(y)\end{array}$ & $\begin{array}{l}\text { Invalid informal } \\
\text { governance }(1-y)\end{array}$ & $\begin{array}{l}\text { Valid informal } \\
\text { governance }(y)\end{array}$ & $\begin{array}{l}\text { Invalid informal governance } \\
\qquad(1-y)\end{array}$ \\
\hline Honest behaviour $(z)$ & $\left(a_{1}, b_{1}, c_{1}\right)$ & $\left(a_{2}, b_{2}, c_{2}\right)$ & $\left(a_{3}, b_{3}, c_{3}\right)$ & $\left(a_{4}, b_{4}, c_{4}\right)$ \\
\hline $\begin{array}{l}\text { Opportunistic } \\
\text { behaviour }(1-z)\end{array}$ & $\left(a_{5}, b_{5}, c_{5}\right)$ & $\left(a_{6}, b_{6}, c_{6}\right)$ & $\left(a_{7}, b_{7}, c_{7}\right)$ & $\left(a_{8}, b_{8}, c_{8}\right)$ \\
\hline
\end{tabular}

TABLe 2: The benefits of the game's three sides.

\begin{tabular}{lccc}
\hline Game strategy & Government & Platform & Enterprises \\
\hline$\left(a_{1}, b_{1}, c_{1}\right)$ & $E_{1}-C_{1}-C_{2}-m-n C_{4}$ & $\lambda_{1} R-C_{3}+m$ & $\lambda_{2} F_{1}-C_{4}+n C_{4}$ \\
$\left(a_{2}, b_{2}, c_{2}\right)$ & $E_{2}-C_{1}-C_{2}-l-n C_{4}$ & $\lambda_{1} l R$ & $F_{2}-C_{4}+n C_{4}$ \\
$\left(a_{3}, b_{3}, c_{3}\right)$ & $E_{1}$ & $\lambda_{1} R-C_{3}$ & $\lambda_{2} F_{3}-C_{4}$ \\
$\left(a_{4}, b_{4}, c_{4}\right)$ & $E_{2}$ & $\lambda_{1} l R$ & $F_{4}-C_{4}$ \\
$\left(a_{5}, b_{5}, c_{5}\right)$ & $E_{3}-C_{1}-C_{2}-m+p$ & $l R$ & $\lambda_{2} F_{1}-w C_{4}-p-q$ \\
$\left(a_{6}, b_{6}, c_{6}\right)$ & $E_{4}-C_{1}-C_{2}+p$ & $R-C_{3}$ & $F_{2}-w C_{4}-p$ \\
$\left(a_{7}, b_{7}, c_{7}\right)$ & $E_{3}$ & $\lambda_{2} F_{3}-w C_{4}-q$ \\
$\left(a_{8}, b_{8}, c_{8}\right)$ & $E_{4}$ & $l R$ & $F_{4}-w C_{4}$ \\
\hline
\end{tabular}

informal governance" strategy is $y$ and the probability of choosing the "invalid informal governance" strategy is $(1-y)$; and suppose the probability that enterprises choose the "honest behaviour" strategy is $z$ and the probability of choosing the "opportunistic behaviour" strategy is $(1-z)$, of which $1>x>0,1>y>0$, and $1>z>0$. The values of $x, y$, and $z$ change in the government's, platform's, and enterprises' constant imitating and learning processes, but the initial value is established.

\section{Modern Analysis}

3.1. Analysis of the Government and Conclusions. The government's expected benefit when it chooses the "valid formal governance" strategy is calculated as

$$
U_{G 1}=y z a_{1}+(1-y) z a_{2}+y(1-z) a_{5}+(1-y)(1-z) a_{6} .
$$

And the government's expected benefit when it chooses the "invalid formal governance" strategy is calculated as

$$
U_{G 2}=y z a_{3}+(1-y) z a_{4}+y(1-z) a_{7}+(1-y)(1-z) a_{8} .
$$

The government's average expected benefit is calculated as

$$
\begin{aligned}
U_{G}= & x U_{G 1}+(1-x) U_{G 2} \\
= & x\left[y z a_{1}+(1-y) z a_{2}+y(1-z) a_{5}+(1-y)(1-z) a_{6}\right] \\
& +(1-x)\left[y z a_{3}+(1-y) z a_{4}+y(1-z) a_{7}+(1-y)(1-z) a_{8}\right],
\end{aligned}
$$

while the government's dynamic replicator equation is

$$
\begin{aligned}
F(x)= & \frac{\mathrm{d} x}{\mathrm{~d} t}=x\left(U_{G 1}-U_{G}\right)=x(x-1) \\
& \cdot\left[C_{1}+C_{2}-p+m y+\left(p+n c_{4}\right) z\right] .
\end{aligned}
$$

Let $z_{0}=\left(p-m y-C_{1}-C_{2}\right) /\left(n C_{4}+p\right)$ :

(1) When $z=z_{0}$, then $F(x) \equiv 0$, in which all equilibrium points are stable.

(2) When $z \neq z_{0}$, let $F(x)=0, x=0$ and $x=1$ are the two equilibrium points.

If we derive $F(x)$ relative to $x$, the outcome is

$$
\frac{\mathrm{d} F(x)}{\mathrm{d} x}=(2 x-1)\left[C_{1}+C_{2}-p+m y+\left(p+n c_{4}\right) z\right] .
$$

There are two cases:

(1) If $z>z_{0}$ when $x=0$, then $(\mathrm{d} F(x) / d x)<0$; when $x=1$, then $(\mathrm{d} F(x) / d x)>0$, and thus, $x=0$ indicates evolutionary stable strategies (ESS).

(2) If $z<z_{0}$, when $x=0$, then $(\mathrm{d} F(x) / d x)>0$; when $x=1$, then $(\mathrm{d} F(x) / d x)<0$, thus $x=1$ is ESS.

This analysis illustrates dynamic evolution in the government, as displayed in Figure 1.

In this figure, the space is divided into two parts with a dashed line. When the government strategy's initial state is in space I, $x=0$ is the ESS, or the point at which formal governance is invalid. When the government strategy's initial state is in Space II, $x=1$ is the ESS, or the point at which formal governance is valid. A further analysis revealed that when the government's incentive to the platform increases, the volume in Space II will decrease and formal governance will tend to be invalid. Similarly, if the government's costs increase, formal governance will be more inclined to be invalid due to implicit cost pressures. 


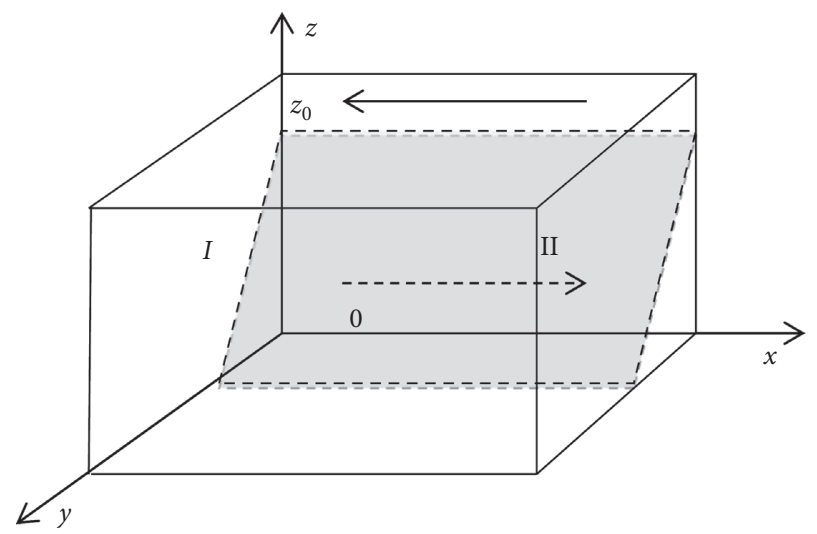

FIgURE 1: The government's dynamic evolutions.

3.2. Analysis of the Platform and Conclusions. The expected benefit to the platform when it chooses the "valid informal governance" strategy is calculated as

$$
U_{p 1}=x z b_{1}+(1-z) x b_{5}+z(1-x) b_{3}+(1-x)(1-z) b_{7} .
$$

while the platform's expected benefit when it chooses the "invalid informal governance" strategy is

$$
U_{p 2}=x z b_{2}+(1-z) x b_{6}+z(1-x) b_{4}+(1-x)(1-z) b_{8} .
$$

The platform's average expected benefit is calculated as

$$
\begin{aligned}
U_{p}= & y U_{p 1}+(1-y) U_{p 2} \\
= & y\left[x z b_{1}+(1-z) x b_{5}+z(1-x) b_{3}+(1-x)(1-z) b_{7}\right] \\
& +(1-x)\left[x z b_{2}+(1-z) x b_{6}+z(1-x) b_{4}+(1-x)(1-z) b_{8}\right] .
\end{aligned}
$$

And the platform's dynamic replicator equation is

$$
\begin{aligned}
F(y)= & \frac{\mathrm{d} y}{\mathrm{~d} t}=y\left(U_{p 1}-U_{p}\right)=y(y-1) \\
& \cdot\left[C_{3}-R+l R-m x+\left(1-\lambda_{1}-l+\lambda_{1} l\right) R z\right] .
\end{aligned}
$$

Let $x_{0}=\left(C_{3}-R+l R+\left(1-\lambda_{1}-l+\lambda_{1} l\right) R z\right) / m$ :

(1) When $x=x_{0}$, then $F(y) \equiv 0$, in that all enterprises' behaviour can be stable.

(2) When $x \neq x_{0}$, let $F(y)=0$, and thus, $y=0$ and $y=1$ are two stable points.

If we derive $F(y)$ relative to $x$, the outcome is

$$
\frac{\mathrm{d} F(y)}{\mathrm{d} y}=(2 y-1)\left[C_{3}-R+l R-m x+\left(1-\lambda_{1}-l+\lambda_{1} l\right) R z\right] .
$$

There are two cases:

(1) If $x>x_{0}$, when $y=0$, then $(\mathrm{d} F(y) / d y)>0$; when $y=1$, then $(\mathrm{d} F(y) / d y)>0$, and thus, $y=1$ is the ESS.

(2) If $x<x_{0}$, when $y=0$, then $(\mathrm{d} F(y) / d y)>0$; when $y=1$, then $(\mathrm{d} F(y) / d y)>0$, and thus, $y=0$ is the ESS.
This analysis indicates the platform's dynamic evolution, as illustrated in Figure 2.

In this figure, the space is divided into two parts by a dashed line. When the platform strategy's initial state is in Space III, $y=0$ is the ESS, and informal governance is invalid at this point. When the platform strategy's initial state is in Space IV, $y=1$ is the ESS, and informal governance is valid at this point. Further analysis indicates that the validity of informal governance relates to the platform's supervisory costs and government subsidies and penalties. When a benefit or government subsidies increase, $x_{0}$ will decrease; thus, the volume of Space IV will increase and informal governance will be valid.

3.3. Analysis of Enterprises and Conclusions. The enterprises' expected benefit when choosing the "honest behaviour" strategy is calculated as

$$
U_{E 1}=x y c_{1}+x(1-y) c_{2}+y(1-x) c_{3}+(1-x)(1-y) c_{4},
$$

while their expected benefit when choosing the "opportunistic behaviour" strategy is

$$
U_{E 2}=x y c_{5}+x(1-y) c_{6}+y(1-x) c_{7}+(1-x)(1-y) c_{8} .
$$

The enterprises' average expected benefit is calculated as

$$
\begin{aligned}
U_{E}= & z U_{E 1}+(1-z) U_{E 2} \\
= & z\left[y c_{1}+x(1-y) c_{2}+y(1-x) c_{3}+(1-x)(1-y) c_{4}\right] \\
& +(1-x)\left[x y c_{5}+x(1-y) c_{6}+y(1-x) c_{7}+(1-x)(1-y) c_{8}\right],
\end{aligned}
$$

while their dynamic replicator equation is

$$
\begin{aligned}
F(z) & =\frac{\mathrm{d} z}{\mathrm{~d} t}=z\left(U_{E 1}-U_{E}\right) \\
& =z(1-z)\left[w C_{4}-C_{4}+q y+\left(p+n C_{4}\right) x\right] .
\end{aligned}
$$

Let $y_{0}=\left(C_{4}-w C_{4}-\left(p+n C_{4}\right) x\right) / q$ :

(1) When $y \equiv y_{0}$, then $F(z) \equiv 0$, in which all can be stable.

(2) When $y \neq y_{0}$, let $F(z)=0, z=0$ and $z=1$ are the two stable points.

We derive $F(y)$ relative to $x$, and the outcome is

$$
\frac{\mathrm{d} F(z)}{\mathrm{d} z}=(1-2 z)\left[w C_{4}-C_{4}+q y+\left(p+n C_{4}\right) x\right]
$$

There are two cases:

(1) If $y>y_{0}, \quad$ when $z=0$, then $(\mathrm{d} F(z) / d z)>0$; when $z=1$, then $(\mathrm{d} F(z) / d z)>0$, and thus, $z=1$ is the ESS. 


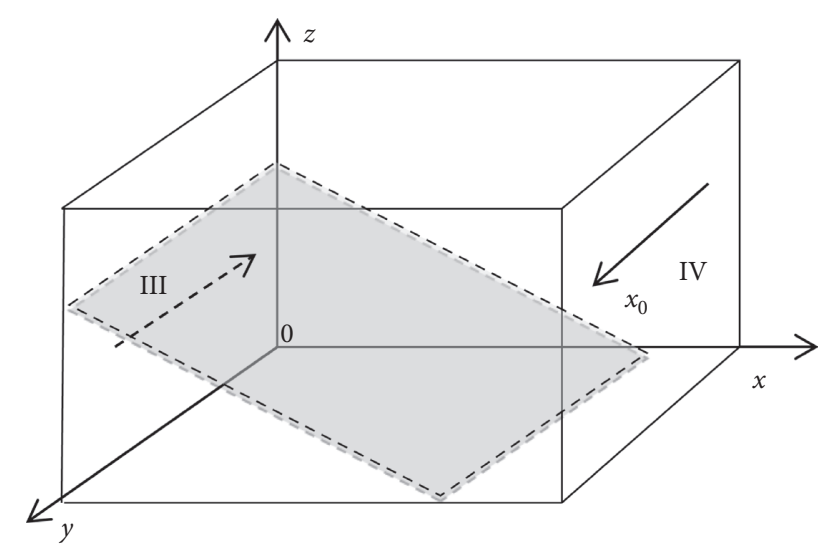

Figure 2: The platform's dynamic evolutions.

(2) If $y<y_{0}$, when $z=0$, then $(\mathrm{d} F(z) / d z)>0$; when $z=1$, then $(\mathrm{d} F(z) / d z)>0$, and thus, $z=0$ is the ESS.

The above analysis reveals the enterprises' dynamic evolution, as displayed in Figure 3.

In this figure, the space is divided into two parts by a dashed line. When the enterprise strategy's initial state is in Space V, $z=0$ is the ESS, and enterprises' behaviour tends toward opportunism at this point. When the enterprise strategy's initial state is in Space VI, $z=1$ is the ESS, and enterprises' behaviour tends toward honesty at this point. Further analysis indicates that enterprises tend to be more honest when the platform's punishment increases, as $y_{0}$ and its volume in Space V will decrease.

This analysis reveals that the subjects of all parties (government, platform, and enterprises) are regarded as one independent. Organic entity integrates Figure 1 through 3; Table 3 displays each space's stability. The tripartite game will not converge to a certain set of stable strategies in a fixed manner, and any change in setting parameters in the dynamic trend may cause the game's players to simultaneously adjust their behavioural strategies. Consequently, no dynamic point exists for the stable state in the integrated model.

Table 3 reveals that both formal and informal governance are valid when the initial state intersects Spaces II, IV, and VI. Furthermore, enterprises will also tend to be honest, as this is the common state in the general platform ecosystem. When the initial state intersects Spaces I, IV, and VI, enterprises will primarily rely on informal governance to constrain such behaviour. Even with a lack of formal governance, enterprises in this situation will be able to maintain appropriate business behaviour. This is the ideal state when developing platform ecosystems.

\section{Numerical Analysis}

Based on the static repeated game in Section 3, a nonlinear characteristic is now introduced to the evolutionary game process among gamers. We further verify the contents of the game analysis by simulating the different situation as follows:

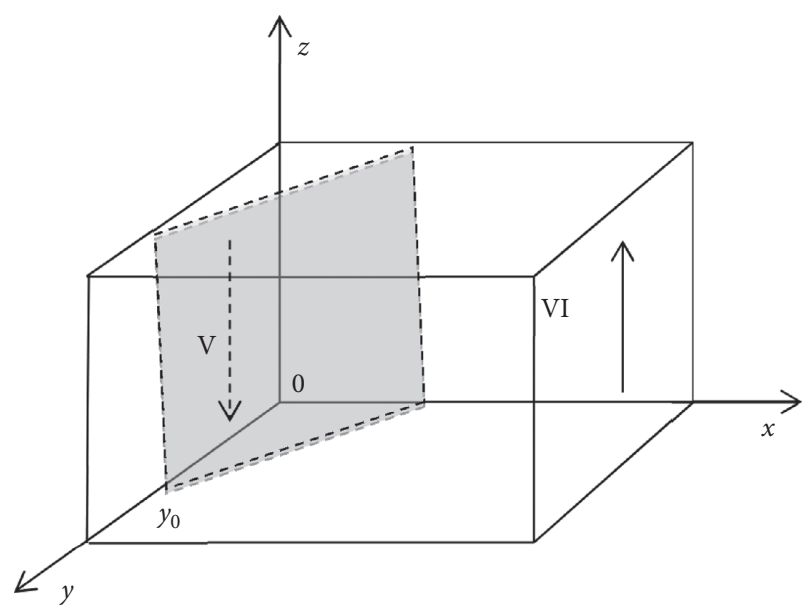

FIgURE 3: The enterprises' dynamic evolutions.

TABle 3: Each subject's strategies in different spaces.

\begin{tabular}{lcccc}
\hline Space & \multicolumn{2}{c}{ I } & \multicolumn{2}{c}{ II } \\
& III & IV & III & IV \\
\hline V & $(0,0,0)$ & $(0,1,0)$ & $(1,0,0)$ & $(1,1,0)$ \\
VI & $(0,0,1)$ & $(0,1,1)$ & $(1,0,1)$ & $(1,1,1)$ \\
\hline
\end{tabular}

$$
\begin{aligned}
C_{1} & =50, \\
C_{2} & =15, \\
C_{3} & =10, \\
C_{4} & =5, \\
F_{1} & =30, \\
F_{2} & =25, \\
F_{3} & =25, \\
F_{4} & =20, \\
R & =100, \\
m & =50, \\
n & =0.8, \\
w & =0.3, \\
p & =100, \\
q & =50, \\
l & =0.5, \\
\lambda_{1} & =1.5, \\
\lambda_{2} & =1.5 .
\end{aligned}
$$

The values of $x_{0}, y_{0}$, and $z_{0}$ are consistent with the following model analysis.

Figure 4 indicates that enterprises gradually behave with good faith given the increase of initial values for formal governance. Thus, enhancing such formal governance is significant in restricting enterprises' behaviours. Initially, formal governance is weaker (initial value $=0.1$ ), and enterprises do not tend to exhibit honest behaviours. However, increasing this initial value more rapidly compels enterprises toward honesty. Therefore, strong formal governance can constrain enterprises' behaviour. 


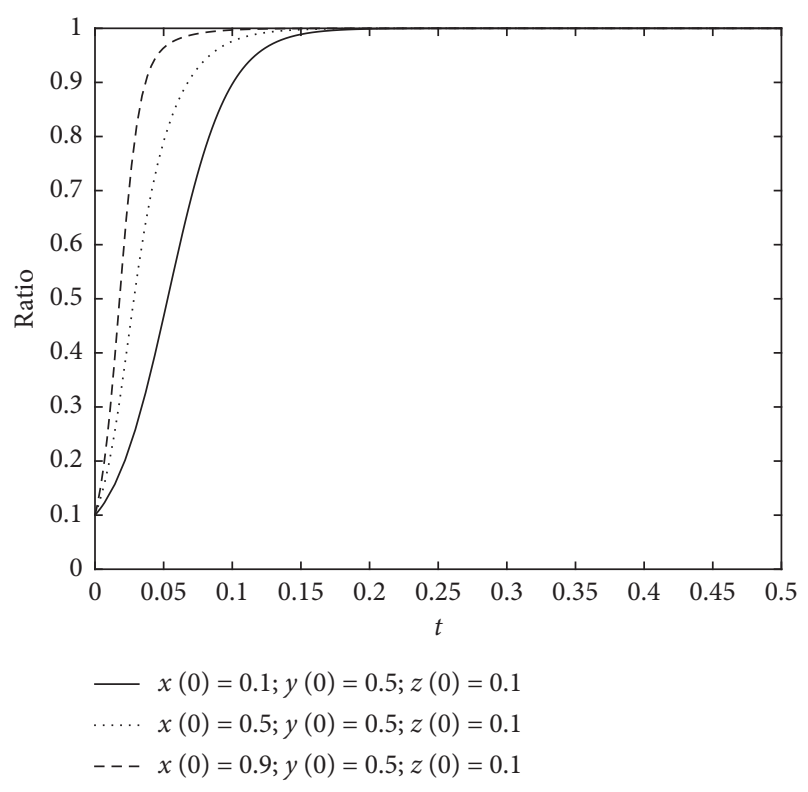

FIGURE 4: Different initial proportions of influence among gamers regarding the evolution of government.

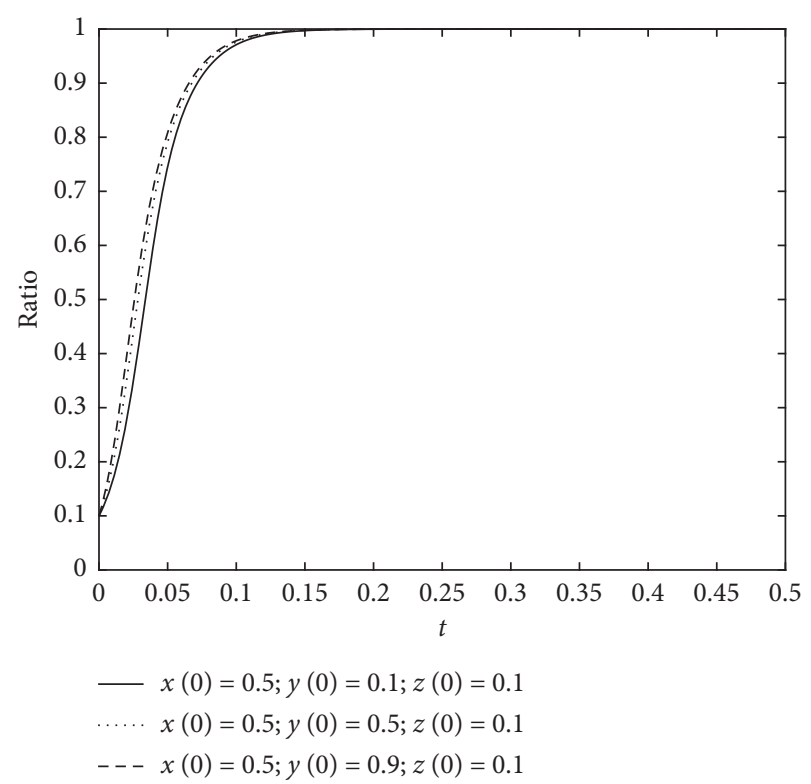

FIGURE 5: Different initial proportions of influence among gamers regarding the evolution of the platform.

Figure 5 demonstrates that enterprises under informal governance gradually tend to become honest. Additionally, when informal governance is initially smaller, enterprises tend to become honest more slowly; with an increase in the initial value for informal governance, this speed increases quickly. Compared with Figure 4, the effect of informal governance on enterprises' behaviours is more stable and moderate, which reveals that increasing the intensity of informal governance has a less drastic impact on enterprises' behaviours than formal governance.

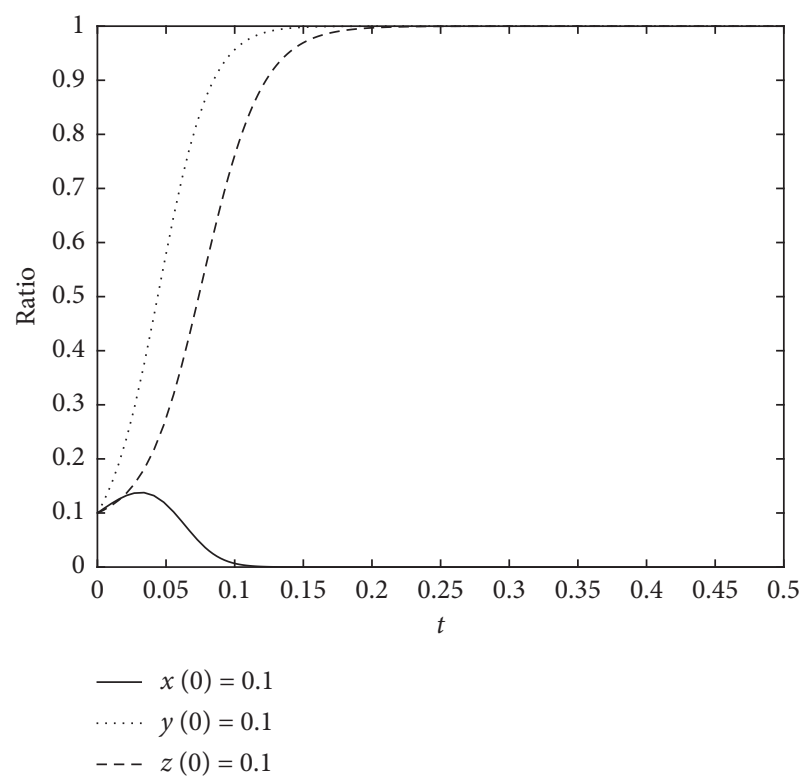

Figure 6: Dynamic evolution process of the game's different players.

Figure 6 depicts the game evolution process that combines formal and informal governance and enterprises' behaviour; in this process, enterprises' behaviour gradually becomes more honest. Formal governance is initially a significant constraint, as the government constantly improves such governance measures to constrain enterprises' behaviour. As time passes, the government will tend to scale back such governance conditions, and informal governance's effects will become more obvious. Regardless of the formal regulatory approach, internal enterprises will ultimately act in good faith without restraint, which verifies the self-organisation of enterprises in the platform ecosystem.

\section{Conclusions}

In the context of a highly globalised economy, SMEs continuously find different alliances to effectively develop and evolve [51]. The platform strategy is one effective way for SMEs to grow [52], although many problems remain in the course of the platform ecosystem's development. For example, one important issue involves effective platform governance to guide the platform's enterprises to develop well. Formal or government-based governance is mandatory and generally relies on the action of governments with the direct power to enforce such codes or laws [53, 54]. Furthermore, informal or platform-based governance is also important in the platform ecosystem [37, 55]. The two governance approaches collectively contribute to the platform ecosystem's self-organisation as enterprises in the platform exhibit fewer opportunistic behaviours but a positive business environment:

(1) In the early stage of developing a platform ecosystem, the government's formal rules and regulations are important in restricting enterprises' behaviour. The government not only bears the responsibility for 
the construction of social infrastructure and public facilities but also formulates corresponding industries, economic policies, and laws suitable for the platform's development, regulates the platform members' self-interest-based behaviours, and corrects the platform enterprises' opportunism. The research results reveal that enterprises under formal or government-based governance tend to exhibit more honest behaviours than under informal or platform-based governance. This may occur because formal governance, such as any relevant laws, are stronger in operation than the faith in a shared vision or adherence to similar cultures. Furthermore, clear laws, systems, and organisational norms can compensate for the shortcomings of informal governance. Therefore, it is essential for the government to supervise and guide enterprises as a platform ecosystem is established. The government should constantly formulate and improve relevant rules and laws, supervise enterprises' behaviour, and provide assistance to the platform ecosystem to build a positive business atmosphere.

(2) Informal or platform-based governance has a stronger impact on enterprises over time than formal or government-based governance. A platform's members may widely accept and closely follow informal governance. The reputation of any enterprises that violate formal regulations will spread rapidly on the platform, and the pressure of public opinion will reposition the offenders at the opposite position of the entire platform, resulting in the loss of customers and partners. The research results demonstrate that formal governance is more restrictive than informal governance, and informal governance is always a stable constraint over time on enterprises' behaviour and is more durable. Formal governance is particularly important at the initial stage of the platform ecosystem's development, although informal governance gradually highlights such constraints as trust and reputation as the platform develops. Therefore, the government should focus on informal or platform-based governance in the platform ecosystem, guide the platform's construction and functions, and emphasise the maintenance and development of mutual trust and a shared vision and culture.

(3) The platform ecosystem is self-organising. Our research reveals that enterprises tend to exhibit honest behaviour under a combination of formal and informal governance. However, companies can also behave in good faith over time, even with a lack of governance. This demonstrates that the platform ecosystem is self-organising. On the one hand, enterprises' behaviour in the platform ecosystem will become increasingly honest by increasing the government's punishment for dishonest enterprises or rewards for honest enterprises. On the other hand, more effective informal governance and stricter punishment mechanisms can also suppress opportunistic behaviour among the platform's enterprises. This can motivate these enterprises to demonstrate their integrity as the ecosystem continues to mature. Therefore, formal and informal governance have been important in not only expanding platform ecosystems but also maintaining the long-term stable development of SMEs and stimulating self-organisation in the platform ecosystem itself.

\section{Data Availability}

The data used to support the findings of this study are included within the article.

\section{Conflicts of Interest}

The authors declare that they have no conflicts of interest.

\section{Acknowledgments}

This work was financially supported by the Fujian Social Science Research Base Major Project (FJ2019JDZ014), National Research Center for the Political Economy of Socialism with Chinese Characteristics (Fujian Normal University) (Q201803), and Chinese National Social Science Foundation of China (19BGL031).

\section{References}

[1] E.-T. Miron, A. Purcarea, and O. Negoita, "Modelling perceived risks associated to the entry of complementors' in platform enterprises: a case study," Sustainability, vol. 10, no. 9, p. 3272, 2018.

[2] K. Karhu, R. Gustafsson, and K. Lyytinen, "Exploiting and defending open digital platforms with boundary resources: android's five platform forks," Information Systems Research, vol. 29, no. 2, pp. 479-497, 2018.

[3] S. Jansen, M. Cusumano, and K. M. Popp, "Managing software platforms and ecosystems," IEEE Software, vol. 36, no. 3, pp. 17-21, 2019.

[4] P. C. Evans and A. Gawer, "The rise of the platform enterprises (the emerging platform economy series no. 1)," 2016, https://www. thecge.net/app/uploads/2016/01/PDF-WEB-Platform-Survey_01_ 12.pdf.

[5] V. Ramaswamy and K. Ozcan, "Brand value co-creation in a digitalized world: an integrative framework and research implications," International Journal of Research in Marketing, vol. 33, no. 1, pp. 93-106, 2016.

[6] M. Magni and L. Maruping, "Unleashing innovation with collaboration platforms," MIT Sloan Management Review, vol. 60 , no. 3, pp. 1-5, 2019.

[7] D. B. Nieborg and A. Helmond, "The political economy of Facebook's platformization in the mobile ecosystem: Facebook Messenger as a platform instance," Media, Culture \& Society, vol. 41, no. 2, pp. 196-218, 2019.

[8] A. Gawer, "Bridging differing perspectives on technological platforms: toward an integrative framework," Research Policy, vol. 43, no. 7, pp. 1239-1249, 2014.

[9] D. P. McIntyre and A. Srinivasan, "Networks, platforms, and strategy: emerging views and next steps," Strategic Management Journal, vol. 38, no. 1, pp. 141-160, 2017. 
[10] S. Mukhopadhyay and H. Bouwman, "Orchestration and governance in digital platform ecosystems: a literature review and trends," Digital Policy, Regulation and Governance, vol. 21, no. 4, pp. 329-351, 2019.

[11] J. Zeng and K. W. Glaister, "Competitive dynamics between multinational enterprises and local internet platform companies in the virtual market in China," British Journal of Management, vol. 27, no. 3, pp. 479-496, 2016.

[12] J. A. Schwarz, "Platform logic: an interdisciplinary approach to the platform-based economy," Policy \& Internet, vol. 9, no. 4, pp. 374-394, 2017.

[13] D. S. Evans and R. Schmalensee, "Markets with two-sided platforms," Issues in Competition and Law and Policy (ABA Section of Antitrust Law), vol. 1, no. 28, pp. 667-693, 2008.

[14] M. Rysman, "The economics of two-sided markets," Journal of Economic Perspectives, vol. 23, no. 3, pp. 125-143, 2009.

[15] C. Lee, D. Lee, and J. Hwang, "Platform openness and the productivity of content providers: a meta-frontier analysis," Telecommunications Policy, vol. 39, no. 7, pp. 553-562, 2015.

[16] P. Spagnoletti, A. Resca, and G. Lee, "A design theory for digital platforms supporting online communities: a multiple case study," Journal of Information Technology, vol. 30, no. 4, pp. 364-380, 2015.

[17] M. de Reuver, C. Sørensen, and R. C. Basole, "The digital platform: a research agenda," Journal of Information Technology, vol. 33, no. 2, pp. 124-135, 2018.

[18] J. F. Moore, "Predators and prey: the new ecology of competition," Harvard Business Review, vol. 71, no. 3, pp. 75-83, 1993.

[19] M. Iansiti and R. Levien, Creating Value in Your Business Ecosystem, pp. 68-78, Harvard Business Review, Boston, MA, USA, 2004.

[20] M. Iansiti and R. Levien, The Keystone Advantage: What New Dynamics of Business Ecosystems Mean for Strategy, Innovation, and Sustainability, Harvard Business School Press, Boston, MA, USA, 2004b.

[21] R. Adner and R. Kapoor, "Value creation in innovation ecosystems: how the structure of technological interdependence affects firm performance in new technology generations," Strategic Management Journal, vol. 31, no. 3, pp. 306-333, 2010.

[22] F. Wei, N. Feng, S. Yang, and Q. Zhao, "A conceptual framework of two-stage partner selection in platform-based innovation ecosystems for servitization," Journal of Cleaner Production, vol. 262, Article ID 121431, 2020.

[23] J. van Dijck, "Governing digital societies: private platforms, public values," Computer Law \& Security Review, vol. 36, Article ID 105377, 2020.

[24] J. Yi, J. He, and L. Yang, "Platform heterogeneity, platform governance and complementors' product performance: an empirical study of the mobile application industry," Frontiers of Business Research in China, vol. 13, no. 1, pp. 1-20, 2019.

[25] N. Helberger, J. Pierson, and T. Poell, "Governing online platforms: from contested to cooperative responsibility," The Information Society, vol. 34, no. 1, pp. 1-14, 2018.

[26] S. Mukhopadhyay, S. Nikou, and H. Bouwman, "Why controls are used in platform ecosystems?" International Journal of E-Services and Mobile Applications, vol. 8, no. 3, pp. 1-19, 2016b.

[27] H. J. Kim, I. Kim, and H. Lee, “Third-party mobile app developers' continued participation in platform-centric ecosystems: an empirical investigation of two different mechanisms," International Journal of Information Management, vol. 36, no. 1, pp. 44-59, 2016.
[28] M. Schreieck, M. Wiesche, and H. Krcmar, "Governing nonprofit platform ecosystems-an information platform for refugees," Information Technology for Development, vol. 23, no. 3, pp. 618-643, 2017.

[29] T. Goldbach and A. Benlian, "Understanding informal control modes on software platforms-the mediating role of thirdparty developers' intrinsic motivation," in Proceedings of the International Conference on Information Systems (ICIS 2015), Fort Worth, TX, USA, December2015.

[30] F. Thies, M. Wessel, and A. Benlian, "Effects of social interaction dynamics on platforms," Journal of Management Information Systems, vol. 33, no. 3, pp. 843-873, 2016.

[31] V. Eloranta and T. Turunen, "Platforms in service-driven manufacturing: leveraging complexity by connecting, sharing, and integrating," Industrial Marketing Management, vol. 55, pp. 178-186, 2016.

[32] The Economic Times, "Govt tightens norms for etailers, bars exclusive deals," 2018, https://economictimes.indiatimes. com/news/economy/policy/government-tighten-norms-for-ecommercecompanies-for-sale-ofproducts/articleshow/67258251. cms.

[33] S. Mukhopadhyay and H. Bouwman, "Multi-actor collaboration in platform-based ecosystem: opportunities and challenges," Journal of Information Technology Case and Application Research, vol. 20, no. 2, pp. 47-54, 2018.

[34] A. Tiwana, B. Konsynski, and A. A. Bush, "Research commentary-platform evolution: coevolution of platform architecture, governance, and environmental dynamics," Information Systems Research, vol. 21, no. 4, pp. 675-687, 2010.

[35] T. Goldbach and V. Kemper, "Should I stay or should I go? The effects of control mechanisms on app developers' intention to stick with a platform," in Proceedings of the 22nd European Conference on Information Systems (ECIS, 2014), AIS Electronic Library (AISeL), Tel Aviv, Israel, June2014.

[36] S. Mukhopadhyay, M. de Reuver, and H. Bouwman, "Effectiveness of control mechanisms in mobile platform ecosystem," Telematics and Informatics, vol. 33, no. 3, pp. 848-859, 2016.

[37] T. Goldbach, A. Benlian, and P. Buxmann, "Differential effects of formal and self control in mobile platform ecosystems: multi-method findings on third-party developers' continuance intentions and application quality," Information \& Management, vol. 55, no. 3, pp. 271-284, 2017.

[38] M. Ceccagnoli, C. Forman, P. Huang, and D. J. Wu, "Cocreation of value in a platform ecosystem! The case of enterprise software," MIS Quarterly, vol. 36, no. 1, pp. 263-290, 2012.

[39] A. Ghazawneh and O. Henfridsson, "Balancing platform control and external contribution in third-party development: the boundary resources model," Information Systems Journal, vol. 23, no. 2, pp. 173-192, 2013.

[40] B. Peng, Y. Wang, S. Zahid, G. Wei, and E. Elahi, "Platform ecological circle for cold chain logistics enterprises: the value co-creation analysis," Industrial Management \& Data Systems, vol. 120, no. 4, pp. 675-691, 2020.

[41] T. L. Huber, T. Kude, and J. Dibbern, "Governance practices in platform ecosystems: navigating tensions between cocreated value and governance costs," Information Systems Research, vol. 28, no. 3, pp. 563-584, 2017.

[42] R. Gorwa, "What is platform governance?" Information, Communication \& Society, vol. 22, no. 6, pp. 854-871, 2019. 
[43] A. Wachhaus, "Platform governance: developing collaborative democracy," Administrative Theory \& Praxis, vol. 39, no. 3, pp. 206-221, 2017.

[44] A. Gawer and M. A. Cusumano, "Industry platforms and ecosystem innovation," Journal of Product Innovation Management, vol. 31, no. 3, pp. 417-433, 2014.

[45] B. Peng, Y. Wang, E. Elahi, and G. Wei, "Behavioral game and simulation analysis of extended producer responsibility system's implementation under environmental regulations," Environmental Science and Pollution Research, vol. 26, no. 17, pp. 17644-17654, 2019.

[46] T. Janowski, E. Estevez, and R. Baguma, "Platform governance for sustainable development: reshaping citizen-administration relationships in the digital age," Government Information Quarterly, vol. 35, no. 4, pp. S1-S16, 2018.

[47] C. J. Martin, P. Upham, and R. Klapper, "Democratising platform governance in the sharing economy: an analytical framework and initial empirical insights," Journal of Cleaner Production, vol. 166, pp. 1395-1406, 2017.

[48] A. Tiwana, "Evolutionary competition in platform ecosystems," Information Systems Research, vol. 26, no. 2, pp. 266-281, 2015.

[49] J. Foerderer, T. Kude, S. W. Schuetz, and A. Heinzl, "Knowledge boundaries in enterprise software platform development: antecedents and consequences for platform governance," Information Systems Journal, vol. 29, no. 1, pp. 119-144, 2019.

[50] B. Peng, Y. Li, E. Elahi, and G. Wei, "Dynamic evolution of ecological carrying capacity based on the ecological footprint theory: a case study of Jiangsu province," Ecological Indicators, vol. 99, pp. 19-26, 2019.

[51] A. Zutshi and A. Grilo, "The emergence of digital platforms: a conceptual platform architecture and impact on industrial engineering," Computers \& Industrial Engineering, vol. 136, pp. 546-555, 2019.

[52] C. Glavas, S. Mathews, and R. Russell-Bennett, "Knowledge acquisition via internet-enabled platforms," International Marketing Review, vol. 36, no. 1, pp. 74-107, 2019.

[53] V. Nash, J. Bright, H. Margetts, and V. Lehdonvirta, "Public policy in the platform society," Policy \& Internet, vol. 9, no. 4, pp. 368-373, 2017.

[54] P. Nooren, N. van Gorp, N. van Eijk, and R. Ó. Fathaigh, "Should we regulate digital platforms? A new framework for evaluating policy options," Policy \& Internet, vol. 10, no. 3, pp. 264-301, 2018.

[55] M. Fenwick, J. A. McCahery, and E. P. M. Vermeulen, "The end of "corporate" governance: hello 'platform' governance," European Business Organization Law Review, vol. 20, no. 1, pp. 171-199, 2019. 